\title{
A SIMPLE PROOF OF A RESULT OF IGLEHART AND SHEDLER
}

\author{
MARK BERMAN, * CSIRO Division of Mathematics and Statistics
}

\begin{abstract}
Iglehart and Shedler (1983) prove that the 'labelled jobs' method for estimation of passage-time characteristics in closed multiclass networks of queues with general service times provides asymptotically shorter confidence intervals than does the 'marked job' method. A simple alternative proof of this result, under slightly more restrictive conditions, is given here.
\end{abstract}

In a recent paper in this journal, Iglehart and Shedler (1983) compare the statistical efficiency of two regenerative simulation methods for the estimation of passage-time characteristics in closed networks of queues. The reader is referred to Section 2 of their paper for a formal definition of the class of queueing networks considered. The basic features of these networks are: $N$ jobs (customers), finite numbers of service centres and job classes with a Markov mechanism for transfer from one (centre, class) pair to another, and mutually independent service times. The Markov transition matrix and the service time distributions are identical for all $N$ jobs. An important consequence of the model assumptions is that

$\left\{P_{n}^{0}: n \geqq 1\right\}$, the sequence of passage times (irrespective of job identity) enumerated in termination order, converges in distribution to a random variable $P^{0}$. Moreover, $P^{0}=P$, the limiting passage time for any marked job. The goal of the simulation is estimation of $r(f)=E\{f(P)\}$, where $f$ is a real-valued (measurable) function.

The two estimators compared by Iglehart and Shedler are both sample means (with random sample size) of the form

$$
\hat{r}^{*}(f)=\sum_{n=1}^{m^{*}(t)} f\left(P_{n}^{*}\right) / m^{*}(t),
$$

where $P_{n}^{*}\left(P_{n_{\mathrm{i}}}^{0}\right.$ in Iglehart and Shedler's notation) is the $n$th completed passage time of type * and $m^{*}(t)$ is the number of $P_{n}^{*}$ completed in $(0, t]$. In the labelled jobs method, $*=0$, i.e. all passage times (irrespective of job identity) are included in the sample mean. In the marked job method, only passage times completed by a given job $i \in(1, \cdots, N)$ are included in the sample mean; in this case, let $*=i, i=1, \cdots, N$. Further details of the methods are given in Sections 3 and 4 of their paper. By embedding both $\left\{P_{n}^{0}: n \geqq 1\right\}$ and $\left\{P_{n}^{i}: n \geqq 1\right\}$ in appropriate regenerative stochastic processes, they show that

$$
t^{\frac{1}{2}}\left\{\hat{r}^{*}(f)-r(f)\right\} / e^{*}(f) \Rightarrow \mathrm{N}(0,1)
$$

Received 8 March 1984; revision received 30 July 1984.

* Postal address: CSIRO Division of Mathematics and Statistics, P.O. Box 218, Lindfield, NSW 2070, Australia. 
as $t \rightarrow \infty$, for $*=0,1, \cdots, N$ (see their Lemma 4.1 ). The quantity $e^{*}(f)$, defined in Lemma (4.1) and Equations (4.5) and (4.7), is of course, proportional to the half-length of the confidence interval of the asymptotic (normal) distribution of the estimator, and is used by Iglehart and Shedler as a measure of the statistical efficiency of $\hat{r}^{*}(f)$. Much of Section 4 of their paper is spent proving that $e^{0}(f) \leqq e^{i}(f), i=1, \ldots, N$, and that the latter quantity is independent of $i \in(1, \cdots, N)$. They remark that the inequality 'is consistent with intuition since the labelled jobs method extracts more passage-time information from a fixed-length simulation run'.

The purpose of this letter is to present a more direct proof (under slightly more restrictive conditions) of the above inequality which the writer believes has a more obvious connection with the intuition mentioned by Iglehart and Shedler. The proof is based on the interpretation of $t^{-\frac{1}{2}} e^{*}(f)$ as the asymptotic standard deviation of $\hat{r}^{*}(f)$ (see Equations (1) and (2)). The mechanism for proving this fact formally is provided by the powerful tool of cumulative processes (Smith (1955), Section 5). Indeed the fundamental Lemma 4.1 of Iglehart and Shedler can also be proved using Smith's Theorem 7 and Corollary 9.1 because, under the conditions of that lemma, the processes $m^{*}(t)$ and $\sum_{n=1}^{m^{*}(t)} f\left(P_{n}^{*}\right)-r(f) m^{*}(t)$ are cumulative with respect to the regenerative processes alluded to above. Applying Smith's Theorem 8 to these two cumulative processes and using mostly the notation of Iglehart and Shedler's Lemma 4.1, we have, under the conditions of that lemma together with the condition $E\left\{\left(\delta_{1}^{*}\right)^{2}\right\}<\infty$, the results:

$$
\begin{gathered}
\lim _{t \rightarrow \infty} E\left\{m^{*}(t)\right\} / t=E\left\{\alpha_{1}^{*}\right\} / E\left\{\delta_{1}^{*}\right\} \equiv \mu^{*}, \\
\lim _{t \rightarrow \infty} \operatorname{Var}\left\{\sum_{n=1}^{m^{*}(t)} f\left(P_{n}^{*}\right)-r(f) m^{*}(t)\right\} / t=\left\{\sigma^{*}(f)\right\}^{2} / E\left\{\delta_{1}^{*}\right\}=V^{*}, \\
e^{*}(f) \equiv\left(E\left\{\delta_{1}^{*}\right\}\right)^{\frac{1}{2}} \sigma^{*}(f) / E\left\{\alpha_{1}^{*}\right\}=\left(V^{*}\right)^{\frac{1}{2}} / \mu^{*} .
\end{gathered}
$$

These results hold for $*=0,1, \cdots, N$. The definition in (5) is just Iglehart and Shedler's definition (4.5) (for $*=0$ ) of (4.7) (for $*=1, \cdots, N$ ). The right-hand side of (5) is (apart from the factor $\left.t^{-\frac{1}{2}}\right)$ just the asymptotic variance of the ratio random variable $\hat{r}^{*}(f)$ (see, for instance, Equation (10.7) in Kendall and Stuart (1977)).

Note also the remark at the bottom of p. 27 of Smith (1955) that, under mild regularity conditions (satisfied by the processes considered here), the results of his Theorem 8 (and hence (3), (4) and (5)) are independent of the initial conditions. It follows from remarks in the first paragraph of this letter than $\mu^{i}, V^{i}$ and hence $e^{i}(f)$ are identical for all $i \in(1, \cdots, n)$. Therefore, we shall henceforth drop the superscript $i$.

Now note that $m^{0}(t)=\sum_{1}^{N} m^{i}(t)$. Hence, from (3)

$$
\mu^{0}=\sum_{i=1}^{N} \mu^{i}=N \mu \text {. }
$$

Let $A^{*}(t)=\sum_{n=1}^{m^{*}(t)} f\left(P_{n}^{*}\right)-r(f) m^{*}(t)$. Noting that $A^{0}(t)=\sum_{1}^{N} A^{i}(t)$, we have

$$
\begin{aligned}
\operatorname{Var}\left\{A^{0}(t)\right\} & =\sum_{i=1}^{N} \operatorname{Var}\left\{A^{i}(t)\right\}+\sum_{i \neq j} \operatorname{Cov}\left\{A^{i}(t), A^{i}(t)\right\} \\
& \leqq \sum_{i=1}^{N} \operatorname{Var}\left\{A^{i}(t)\right\}+\sum_{i \neq j}\left[\operatorname{Var}\left\{A^{i}(t)\right\} \operatorname{Var}\left\{A^{i}(t)\right\}\right]^{\frac{1}{2}} \\
& =\left[\sum_{i=1}^{N}\left[\operatorname{Var}\left\{A^{i}(t)\right\}\right]^{\frac{1}{2}}\right]^{2} .
\end{aligned}
$$


Dividing both sides of (7) by $t$, letting $t \rightarrow \infty$ and using (4), one obtains

$$
V^{0} \leqq\left\{\sum_{i=1}^{N}\left(V^{i}\right)^{\frac{1}{2}}\right\}^{2}=N^{2} V
$$

It follows immediately from (5), (6) and (8) that $e^{0}(f) \leqq e(f)$.

\section{References}

IgLeHART, D. L. AND SHEDLER, G. S. (1983) Statistical efficiency of regenerative simulation methods for networks of queues. Adv. Appl. Prob. 15, 183-197.

Kendall, M. G. AND Stuart, A. (1977) The Advanced Theory of Statistics, Vol. 1, 4th edn. Griffin, London.

SmITH, W. L. (1955) Regenerative stochastic processes. Proc. R. Soc. London A 232, 6-31. 